\title{
Fabrication and Characterization of Inverted Silicon Pyramidal Arrays with Randomly Distributed Nanoholes
}

\author{
Yue Zhao ${ }^{1,2}$, Kaiping Zhang ${ }^{1}$, Hailiang $\mathrm{Li}^{1, *}$ and Changqing Xie ${ }^{1}$ \\ 1 Key Laboratory of Microelectronic Devices \& Integrated Technology, Institute of Microelectronics of Chinese \\ Academy of Sciences, Beijing 100029, China; zhaoyue@ime.ac.cn (Y.Z.); zhangkaiping@ime.ac.cn (K.Z.); \\ xiechangqing@ime.ac.cn (C.X.) \\ 2 University of Chinese Academy of Sciences, Beijing 100049, China \\ * Correspondence: lihailiang@ime.ac.cn
}

Citation: Zhao, Y.; Zhang, K.; Li, H.; Xie, C. Fabrication and

Characterization of Inverted Silicon

Pyramidal Arrays with Randomly

Distributed Nanoholes.

Micromachines 2021, 12, 931.

https://doi.org/10.3390/

mi12080931

Academic Editor: Lucia Romano

Received: 12 July 2021

Accepted: 3 August 2021

Published: 5 August 2021

Publisher's Note: MDPI stays neutral with regard to jurisdictional claims in published maps and institutional affiliations.

Copyright: (c) 2021 by the authors. Licensee MDPI, Basel, Switzerland. This article is an open access article distributed under the terms and conditions of the Creative Commons Attribution (CC BY) license (https:// creativecommons.org/licenses/by/ $4.0 /)$.

\begin{abstract}
We report the fabrication, electromagnetic simulation and measurement of inverted silicon pyramidal arrays with randomly distributed nanoholes that act as an anti-reflectivity coating. The fabrication route combines the advantages of anisotropic wet etching and metal-assisted chemical etching. The former is employed to form inverted silicon pyramid arrays, while the latter is used to generate randomly distributed nanoholes on the surface and sidewalls of the generated inverted silicon pyramidal arrays. We demonstrate, numerically and experimentally, that such a structure facilitates the multiple reflection and absorption of photons. The resulting nanostructure can achieve the lowest reflectance of $0.45 \%$ at $700 \mathrm{~nm}$ and the highest reflectance of $5.86 \%$ at $2402 \mathrm{~nm}$. The average reflectance in the UV region (250-400 nm), visible region $(400-760 \mathrm{~nm})$ and NIR region $(760-2600 \mathrm{~nm})$ are $1.11,0.63$ and $3.76 \%$, respectively. The reflectance at broadband wavelength $(250-2600 \mathrm{~nm})$ is 14.4 and 3.4 times lower than silicon wafer and silicon pyramids. In particular, such a structure exhibits high hydrophobicity with a contact angle up to $132.4^{\circ}$. Our method is compatible with well-established silicon planar processes and is promising for practical applications of anti-reflectivity coating.
\end{abstract}

Keywords: anti-reflectivity; broadband; metal-assisted chemical etching; anisotropic wet etching

\section{Introduction}

Anti-reflectivity coating plays a key role in the development of modern optoelectronic devices and has found various interesting applications. For example, anti-reflectivity coating is highly desirable to improve the quantum efficiency of back-illuminated detectors [1], to reduce the optical losses of solar cells [2], to facilitate the efficient capture of near infrared light in night vision cameras [3], and to create additional electron-hole pairs in avalanche photodiodes [4,5]. Generally, there are two categories to suppress reflection [6], i.e., enhancing transmission and enhancing absorption. To realize enhanced transmission, coatings that rely on the thin-film interference are adopted. The thickness of the layer should be a quarter or half of the wavelength of the light. Considering the minimum reflectivity under the wide-range light spectrum, the introduction of a multilayer structure can improve performance. However, the design and manufacture process are complicated. Additionally, most of these anti-reflective coating solutions are expensive because they require vacuum technology, which could be problematic in mass production. To realize the enhanced absorption, micro-nano structures are employed to change the direction of light to reduce the reflection, and materials with special optical properties such as $\mathrm{Si}_{3} \mathrm{~N}_{4}$ [7], $\mathrm{SiO}_{2}$ [8], $\mathrm{TiO}_{2}[9,10]$ and GaAs [11] can interact with photons. The micro-nano structures that realize surface anti-reflection have various forms, most of the structures are derived from the moth-eye structure that was first discovered by Bernhard in nature [12,13]. Based on the moth-eye structure, a series of anti-reflective micro-nano structures have been derived, such as nano-pillars [14], nano-cones [15], nano-holes [16], nano-wires [17,18], etc. [19,20]. 
There are the following two main methods to fabricate: (i) dry etching, including reactive ion etching (RIE) and chemically assisted ion beam etching [17]; and (ii) wet etching, which uses chemical etchant solutions such as alkaline and acidic solutions [21,22].

Silicon is one of the most important semiconductor materials for energy conversion due to its photovoltaic effect $[2,16,23]$. However, incident light cannot be completely reflected [24]. Light-trapping films can improve the absorption of their composite devices by modifying its surface microstructure in the wavelength range of 300-1100 $\mathrm{nm}[17,25,26]$. Compared to bare silicon or silicon with other structures, silicon with pyramidal structures can have reduced reflectance and a simple and stable fabrication process [21,27]. Anisotropic wet etching [28] takes advantage of the fact that different crystal orientations of $\mathrm{Si}$ have different etching rates in alkaline solutions, and, thus, allows for the preparation of large-area micro-nano pyramidal array structures on the surface of monocrystalline silicon. In conventional structures, pyramids are randomly distributed and overlap each other [20,29], which affect the efficiency of reflection. When the light sources impinge the wafer, the reflected light path is confirmed because all the reflecting surfaces are at the same angle [28]. In other words, the conventional pyramidal structure has a limited light trapping performance, and silicon with a regular inverted pyramidal textured film applied can achieve a reflectance of about 14\% [30]. The principle of Metal-assisted chemical etching (MacEtch) is simple [31-33], Au nano particles (NPs) act as catalysts to induce local oxidation in acidic solutions, forming pores underneath. The corrosion rate of silicon under $\mathrm{Au}$ NPs is much higher than that of uncovered silicon in a mixed solution composed of hydrogen peroxide and hydrofluoric acid [34]. As the Au NPs sink, porous or columnar silicon nanostructures are formed. MacEtch can be used to generate deep structures, such as nanowires or pore structures, with a large aspect ratio [35]. Vertically aligned arrays of silicon nanostructures can provide nearly ideal photon absorption, thus reducing the size of semiconductor absorbers [16]. However, there is still the problem of a single direction of the reflected light path.

Here, an inverted pyramidal array with deep etched holes on a silicon wafer is realized by designing a fabrication process that combines the advantages of both etching methods. After conventional lithography on the silicon substrate, anisotropic wet etching in a $\mathrm{KOH}$ solution is performed to form the inverted pyramidal array. The random distribution of holes on the surface increases the structure density and aspect ratio, and this structure facilitates multiple reflection and absorption of light, thus improving the reflection efficiency. Since the intrinsic band gap of silicon is $1.12 \mathrm{eV}$, the general black silicon mainly concentrates on suppressing the reflection of light at wavelengths below $1100 \mathrm{~nm}$ [36], and the above structure ensures low reflectance in the visible region. Therefore, this structure can achieve a large surface area via a simple method with no toxic and harmful by-products and has good compatibility with the well-established silicon planar processes.

The reflectance is related to the permittivities at the surface; in 1904, Maxwell Garnett developed a simple but immensely successful homogenization theory. As any such theory, it aims to approximate a complex electromagnetic medium such as a colloidal solution of gold micro-particles in water with a homogeneous effective medium. The Maxwell Garnett mixing formula gives the permittivity of this effective medium in terms of the permittivities and volume fractions of the individual constituents of the complex medium. For antireflection structures such as nanostructures and pyramids, corresponding optical models can be established for auxiliary analysis through the effective medium analysis [37]. For the anti-reflection structure, the effective medium analysis regards the entire anti-reflection structure as a combination of multiple media. The Maxwell Garnett theory starts from the macroscopic Maxwell's equations, which are assumed to be valid on a fine scale inside the composite.

\section{Materials and Manufacturing Methods}

The process flow for fabricating inverted pyramidal arrays with deep etched holes is shown in Figure 1. In our experiments, a 4-inch n-Type $<100>$ wafer with 100-nanometer 
silicon oxide was used as substrate. The photoresist NR1500 was spin-coated onto the $\mathrm{SiO}_{2}$ with a thickness of $300 \mathrm{~nm}$ and then baked on a hot plate at $180^{\circ} \mathrm{C}$ for $2 \mathrm{~min}$ (Figure 1a). The photoresist was exposed with a photolithograph (Suss MA6, Karl Suss, Germany) for pattern definition (Figure 1b) to form a square array. Reactive ion etching (RIE) was performed to transfer the resist pattern to the $\mathrm{SiO}_{2}$ layer, forming an $\mathrm{Si}$ square array exposed on the surface (Figure 1c).

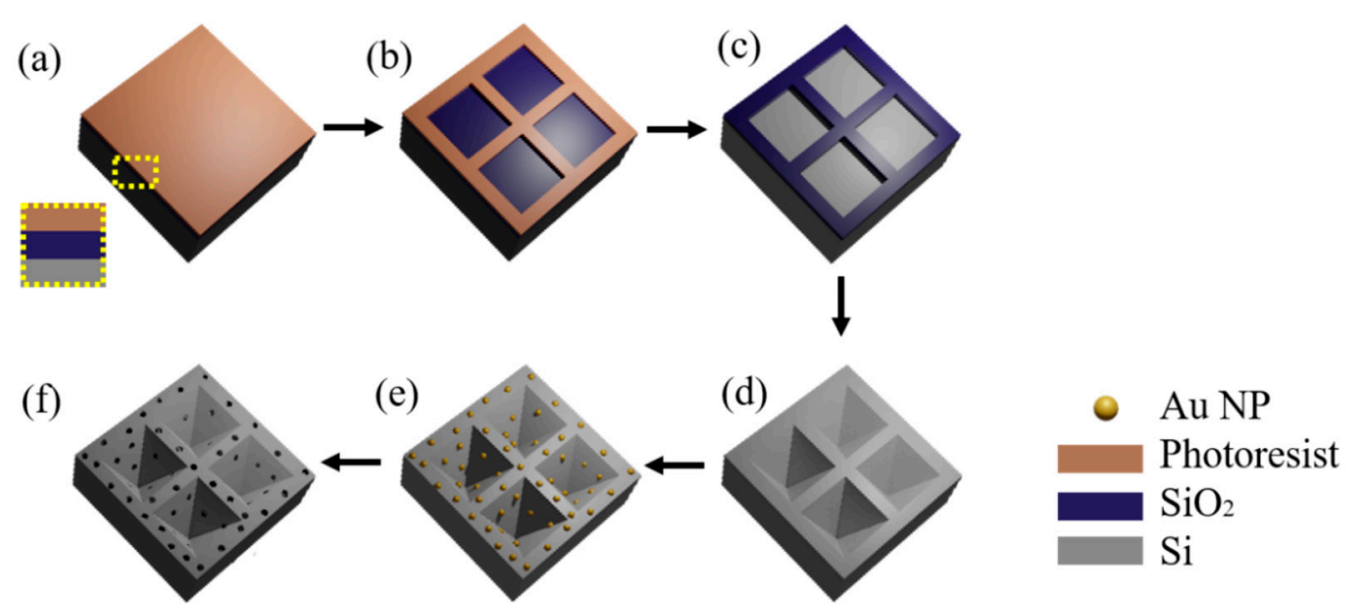

Figure 1. Schematic diagram of an inverted pyramidal array with deep etched holes using anisotropic wet etching and MacEtch process. (a) Oxidized silicon wafers substrate with photoresist NR1500; (b) Photo lithography; (c) Reactive ion etching (RIE) etching and remove the photoresist; (d) Anisotropic wet etching; (e) Deposition of Au layer and annealed to obtain random Au NPs; (f) MacEtch process.

An anisotropic wet etching process was used to generate an inverted pyramidal structure on (100) planar silicon (Figure 1d), which was etched at $85^{\circ} \mathrm{C}$ in a solution containing DI water and $\mathrm{KOH}(30 \%)$, and the size of the resultant pyramid was determined by the etching time. For example, the etching time for a pyramid with side length of $6 \mu \mathrm{m}$ in an alkaline etching solution is about $20 \mathrm{~min}$. After etching, $\mathrm{SiO}_{2}$ was removed with a $10 \%$ HF solution.

A 4-nanometer $\mathrm{Au}$ thin film was deposited on the surface by a magnetron sputtering system (ACS-4000, ULVAC Company, Kanagawa, Japan). The working pressure was maintained at $4.5 \times 10^{-6}$ Torr, and the temperature of the chamber was kept at $25^{\circ} \mathrm{C}$ during the deposition process. After annealing at $300{ }^{\circ} \mathrm{C}$ for $3 \mathrm{~min}$ in air, Au NPs are obtained randomly on the sidewall of the inverted pyramid cavity and the surface of the silicon wafer with an average size of $6 \mathrm{~nm}$ (Figure 1e).

Then, MacEtch process was carried out to generate etching holes on the surface (Figure 1f). At room temperature, MacEtch takes place in $10 \mathrm{~mL}$ of hydrogen peroxide $\left(\mathrm{H}_{2} \mathrm{O}_{2}, 30 \%\right), 10 \mathrm{~mL}$ of Hydrofluoric acid (HF, $\left.49 \%\right)$ and $100 \mathrm{~mL}$ of DI water. Additionally, the depth of the corrosion hole is related to the etching time.

\section{Results and Discussion}

The silicon structure of the inverted pyramidal array after anisotropic wet etching was observed using scanning electron microscopy (SEM) (ZEISS, SUPRA-55, Oberkochen, Germany). Figure 2a shows the SEM image of the top view of the silicon pyramidal array. The inverted pyramidal array has a period of $8 \mu \mathrm{m}$ and a side length of $6 \mu \mathrm{m}$. The corresponding cross-section SEM image of the silicon pyramidal array is shown in Figure $2 \mathrm{~b}$. The angle between the sidewall and the bottom surface is $54.74^{\circ}$ (the angle between the (111) plane and the (100) plane of monocrystalline $\mathrm{Si}$ ) and the depth is $4.1 \mu \mathrm{m}$. In order to observe the whole pyramid and the Au NPs of such a small size at the same time, we take a pyramid with a side length of $2 \mu \mathrm{m}$ as an example. Figure $3 \mathrm{a}$ shows that 
Au NPs are randomly distributed on the surface of silicon after annealing, with an average size of $6 \mathrm{~nm}$. Figure $3 \mathrm{~b}$ is zoomed in view of Au NPs at selected positions in Figure 3a.
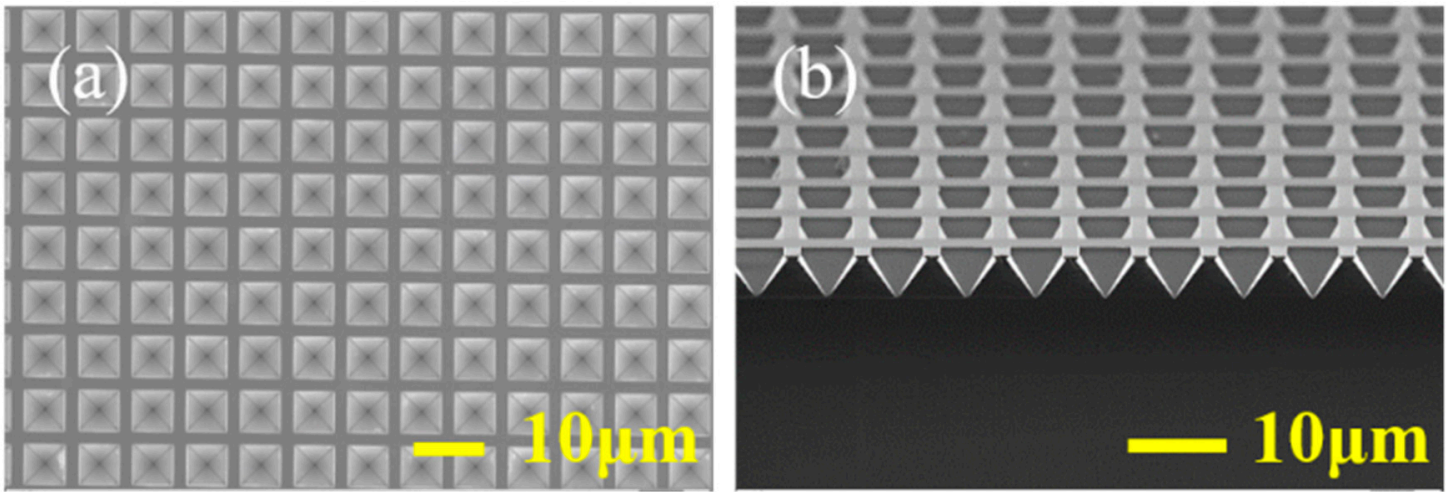

Figure 2. SEM images of the inverted pyramidal array images using anisotropic wet etching (a) top view, (b) cross section view.
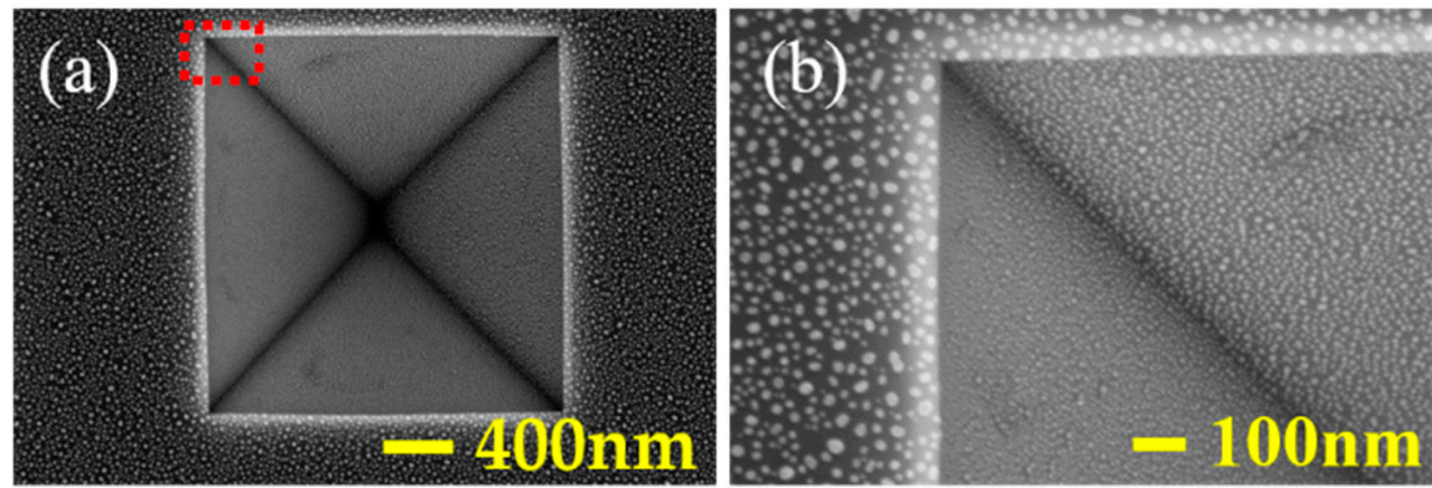

Figure 3. SEM images of randomly distributed Au NPs after annealing (a) top view, (b) zoomed in view of Au NPs at selected positions in (a).

We measured the reflectance of the as-prepared samples with a UV-vis-NIR Spectrophotometer (SHIMADZU, UV3600plus) equipped with an integrating sphere with 2.0-nanometer spectral bandwidth. The inner diameter of the integrating sphere is $60 \mathrm{~mm}$, and the inner layer material is $\mathrm{BaSO}_{4}$. The light measuring range is $250-2600 \mathrm{~nm}$. Before testing the samples, we used $\mathrm{BaSO}_{4}$ as a white board to perform the baseline calibration. After that, the sample is fixed at the corresponding position, and the diffuse reflection is performed. Indeed, there was a tiny amount of transmitted NIR light reflected and diffused back into the integrating sphere. It was not considered in this article. We measured the reflectance of annealed inverted pyramidal arrays with side lengths of 3, 4, 5 and $6 \mu \mathrm{m}$, respectively. The corresponding periods are 5, 6, 7 and $8 \mu \mathrm{m}$, respectively (Figure 4a). The reflectance decreases slightly as the size increases with a wavelength below $1100 \mathrm{~nm}$. Since the angle of the etched surface is fixed, the depth will increase as the size of the inverted pyramid increases, the surface area will be larger, and the number of Au NPs will be more. Light will be trapped more efficiently in larger size pyramids. This morphology ensures the low reflectance, which could be attributed to gradient refractive index and multiple scattering effects. However, there is an increase in the NIR region near $1100 \mathrm{~nm}$. This phenomenon is caused by the intrinsic bandgap of silicon $(1.12 \mathrm{eV})$. There is no obvious regularity on the reflectance over $1100 \mathrm{~nm}$ of different sizes of the inverted pyramids. The average reflectance (250-2600 $\mathrm{nm}$ ) of the pyramidal arrays with four different sizes are 13.6, $12.4,11.4$ and $10.5 \%$. As we can see the average reflectance decreases slightly as the size increases over $1100 \mathrm{~nm}$. We also measured the reflectance of polished Si wafer (Figure 4c) as a control, but samples with smooth surfaces such as mirrors and glass have almost no 
diffuse reflection. As the diffuse reflection is almost $0 \%$, polished Si wafer is suitable for measurement in specular reflection mode. The average reflectance of polished Si wafer at broadband wavelength $(250-2600 \mathrm{~nm})$ is $44.6 \%$.
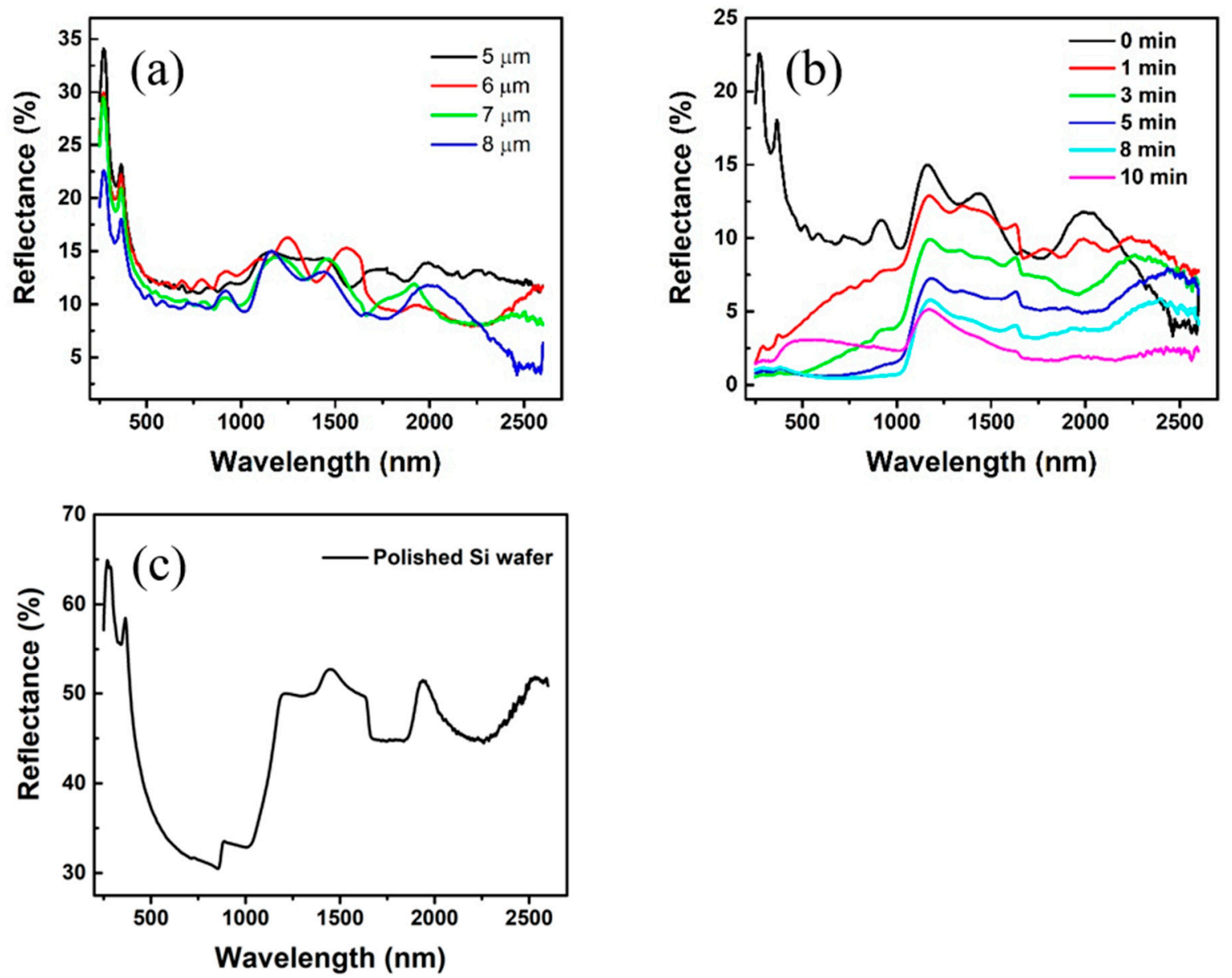

Figure 4. Reflectance spectra from $250 \mathrm{~nm}$ to $2600 \mathrm{~nm}$ for annealed inverted pyramidal arrays (a) with periods of 5, 6, 7 and $8 \mu \mathrm{m}$ before MacEtch process; (b) with period of $8 \mu \mathrm{m}$ treated by the MacEtch processes for 0, 1, 3, 5, 8, 10 min; (c) Specular reflection for polished Si wafer from $250 \mathrm{~nm}$ to $2600 \mathrm{~nm}$.

We take the sample with an 8-micrometer period as an example: after the inverted pyramidal array with Au NPs were immersed in the etching solution, we observed the resultant morphology of inverted pyramids with different treating times of MacEtch (i.e., 3,8 and $10 \mathrm{~min}$, Figure $5 \mathrm{a}-\mathrm{c}$ ). As the etching time increases, both the etching depth and the surface roughness increase. It can be observed directly that the surface of the wafer gradually loses its luster and becomes darker due to the larger photon absorption. From 10 min onwards, the surface of the wafer becomes a grayish white. As the time increases, the etched holes are merged due to continuous expansion, the gap between the two adjacent inverted pyramids decreases. In this way, the reflection efficiency begins to decrease (Figure $4 \mathrm{~b}$ ). It can be inferred that the holes produced by MacEtch have made a great contribution to reducing reflectance. The anti-reflection performance in the NIR region (1100-2600 $\mathrm{nm}$ ) is improved after MacEtch, with an average reflectance decreased from 10.5 to $4.40 \%$ at $8 \mathrm{~min}$ and $3.1 \%$ at $10 \mathrm{~min}$. Taking a sample treated with $8 \mathrm{~min}$ MacEtch as an example, it can be seen from the top view, side view and enlarged view (Figure $5 d-f$ ) that the etched holes are almost perpendicular to the surface of the silicon substrate, and the average depth is $200 \mathrm{~nm}$. The average reflectance at broadband wavelength $(250-2600 \mathrm{~nm})$ is $3.1 \%$, which is 14.4 times lower than that of the polished Si wafer. As shown in Figure $5 \mathrm{~g}$, the method can be simply applied to a wafer-level fabrication of inverted silicon pyramidal 
arrays with randomly distributed nanoholes with a high wafer yield of Si anti-reflectivity coating. This demonstration indicates that our method is an effective way for the mass production of an Si anti-reflectivity coating with excellent anti-reflectivity performance.
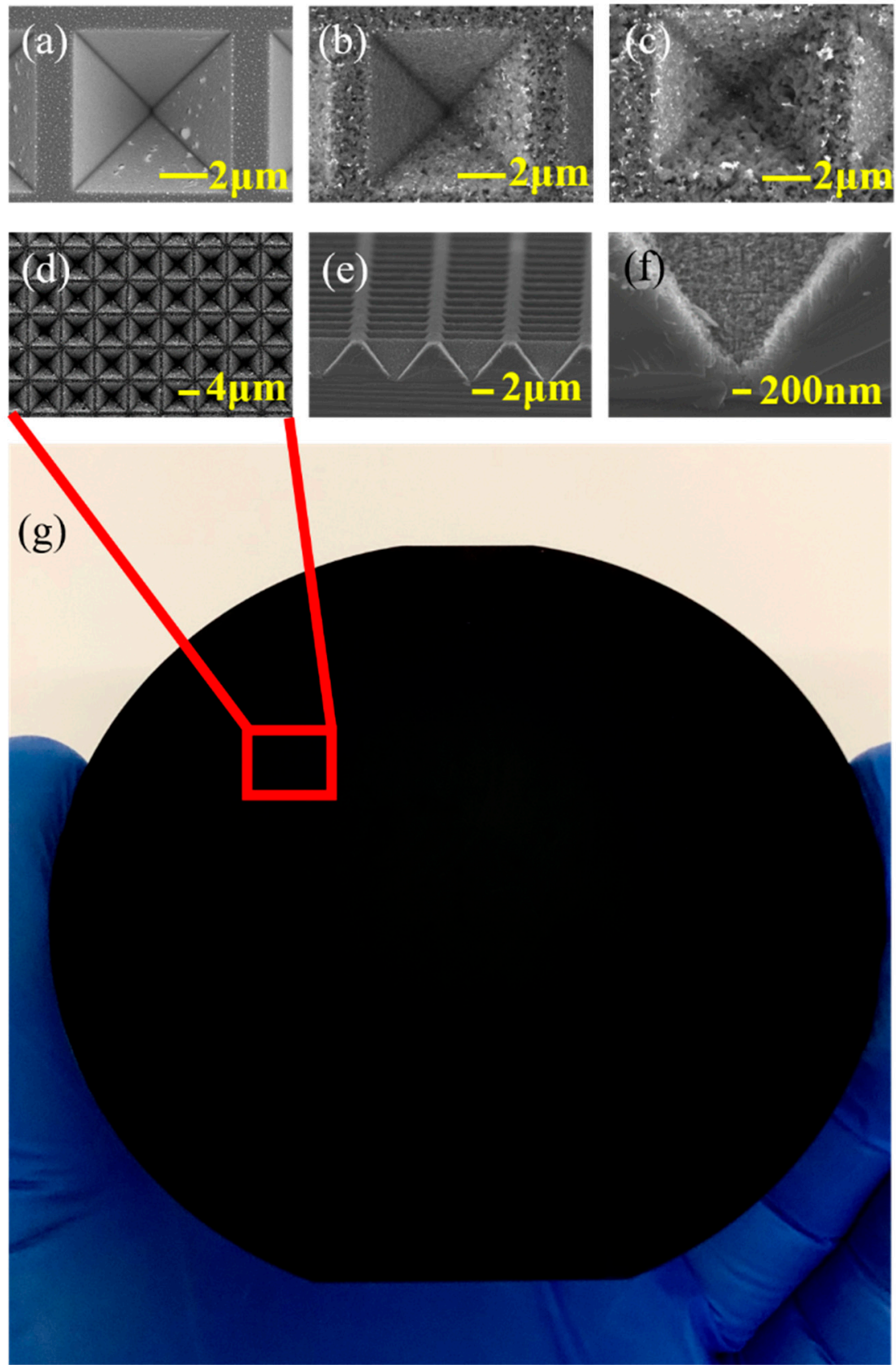

Figure 5. SEM images of inverted pyramids with period of $8 \mu \mathrm{m}$ treated by the MacEtch processes for (a) $3 \mathrm{~min}$; (b) $8 \mathrm{~min}$; (c) $10 \mathrm{~min}$; SEM micrographs of (d) top view; (e) side view; (f) enlarged view of inverted pyramids with period of $8 \mu \mathrm{m}$ treated by the MacEtch processes for $8 \mathrm{~min}$; (g) wafer-level fabrication of inverted silicon pyramidal arrays with randomly distributed nanoholes with a high wafer yield of Si anti-reflectivity coating.

In order to explain this phenomenon, we refer to the Maxwell Garnett model. Nanoscale surface structures, such as nanowire, nanorod and nano cone, provide enhanced absorption properties via anti-reflective and light scattering effects and offer a large junction area for charge separation and radial junction architecture. The further increase in the number of small structures on the side walls reduces the reflectance, demonstrating the efficient 
light trapping performance of the hierarchical structure. It is well known that the working principle of a superior light trapping structure is to efficiently complement the mismatch of the refractive index between the air and the bulk silicon, while for the hierarchical structure, since relatively small structures exist on the side walls, the mismatch between air and silicon obtain a better improvement and, thus, an improved gradual change of the refractive index profile occurs. Therefore, the hierarchical structure can provide a much lower reflectance than that of the other structures [37]. The Maxwell Garnett model regards the silicon material as the main material of the composite $\varepsilon_{m}$, the air in the gap as the filling material $\varepsilon_{i}$ and the effective permittivity $\varepsilon_{e f f}$ of the composite can be calculated using the following equation according to Maxwell Garnett mixing formula [38]:

$$
\frac{\varepsilon_{e f f}-\varepsilon_{m}}{\varepsilon_{e f f}+\varepsilon_{m}}=\delta_{i} \frac{\varepsilon_{i}-\varepsilon_{m}}{\varepsilon_{i}+2 \varepsilon_{m}}
$$

where $\varepsilon_{e f f}, \varepsilon_{m}$ and $\varepsilon_{i}$ are the effective permittivity of the composite, the permittivity of the primary material and the permittivity of the secondary material and $\delta_{i}$ is the volume fraction of the secondary material in the composite.

According to the wave equation, Equation (2) shows that the reflectance is related to the permittivity of the media at the boundary as follows:

$$
R=\left|\frac{\sqrt{\varepsilon_{e f f}}-1}{\sqrt{\varepsilon_{e f f}}+1}\right|^{2}
$$

According to Maxwell's equations, light intensity is proportional to the square of the electric field intensity; therefore, the electric field intensity can explain the distribution of the light. We used FDTD to simulate the electromagnetic enhancement phenomenon and observe the internal electric field. To simulate the electric field intensity distribution, we built an inverted pyramid to simplify the model. We simulated the electric field distribution at 250-2600 nm in the following three situations: (1) an inverted pyramid without AuNPs; (2) an inverted pyramid with AuNPs; (3) an inverted pyramid with nanoholes and AuNPs; (4) an inverted pyramid with nanoholes but without AuNPs. The schematics are in the first column in Figure 6. The black frame in the schematic diagram is the simulation area, the gray part is $\mathrm{Si}$, the white part is air, and the golden part is AuNP. The boundary conditions in the $x$ and $y$ directions were applied to periodic boundary conditions. The perfect matching layer was applied in the $\mathrm{z}$ direction. The incident light was set as a plane wave along the $z$ direction. As shown in Figure $6 \mathrm{~b}, \mathrm{~d}, \mathrm{f}$, the distribution plot of the cross-section view show that "hot spots" were formed in the inverted pyramid cavity at the wavelength $\lambda=303,608$ and $1268 \mathrm{~nm}$, which were selected from UV, visible and NIR regions, respectively. The side length of the pyramid is $2 \mu \mathrm{m}$, the diameter of the AuNPs is $10 \mathrm{~nm}$ and the depth of the holes is $200 \mathrm{~nm}$. The electromagnetic field in the concave structure is higher than the surrounding $\mathrm{Si}$. It demonstrated that the distribution of light (electromagnetic waves) is greatly influenced by the designed structure. 


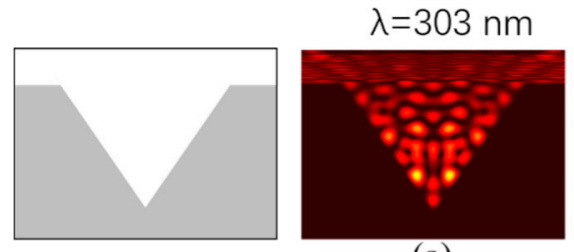

(a)
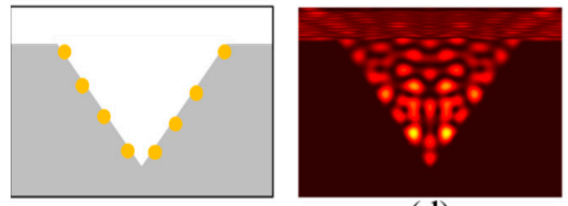

(d)
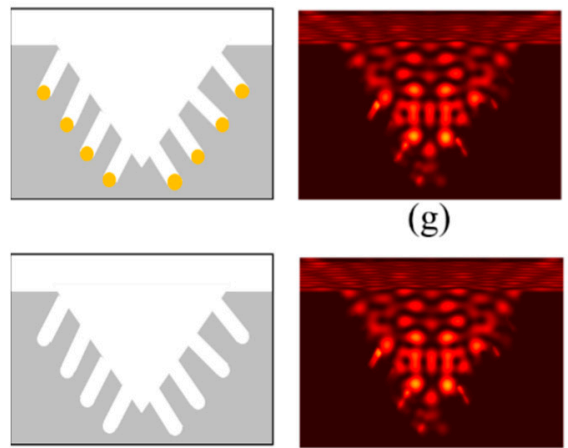

(g)

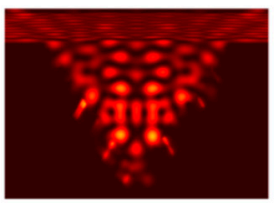

(j)

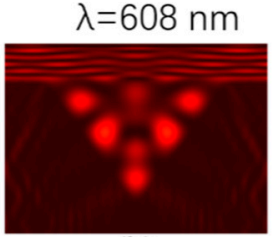

(b)

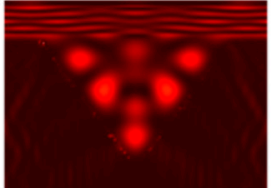

(e)

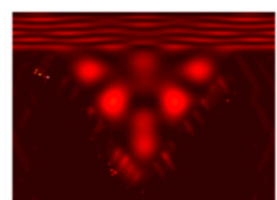

(h)

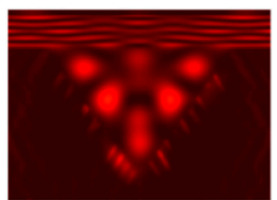

(k)

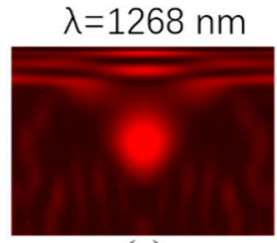

(c)

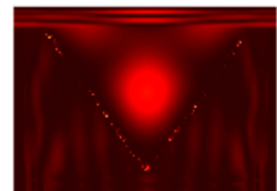

(f)

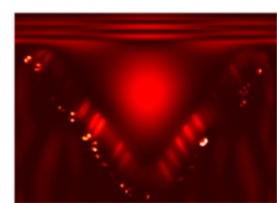

(i)

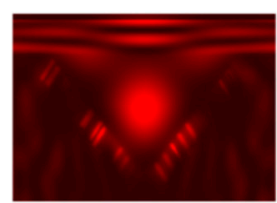

(1)

Figure 6. FDTD simulation of $\lambda=303,608,1268 \mathrm{~nm}$ with corresponding morphologies. (a-c) Simulation of an inverted pyramid without AuNPs at $\lambda=303,608,1268 \mathrm{~nm}$; (d-f) Simulation of an inverted pyramid with AuNPs at $\lambda=303,608,1268 \mathrm{~nm}$; (g-i) Simulation of an inverted pyramid with nanoholes and AuNPs at $\lambda=303,608,1268 \mathrm{~nm} ;(\mathbf{j}-\mathbf{l})$ Simulation of an inverted pyramid with nanoholes but without AuNPs at $\lambda=303,608,1268 \mathrm{~nm}$.

At the same time, the reflected or scattered light is weakened again on the other sidewalls through the interaction with Au NPs on the surface. We add several Au NPs on the surface of the inverted pyramid in the model in Figure $6 \mathrm{~d}-\mathrm{f}$. Compared with Figure $6 \mathrm{a}-\mathrm{c}$, a V shape electric field intensity enhancement can be observed, which were the same shape as the surface of inverted pyramids. Compared with Figure 6a-f, Figure $6 \mathrm{~h}-\mathrm{g}$ proved that it is indeed the holes produced by the AuNPs that have an obvious antireflection effect. From the comparison of Figure $6 \mathrm{~h}-\mathrm{g}$, we find that the electric field of AuNPs are getting stronger and stronger as the wavelength increases. Therefore, the electric field was enhanced on the surface caused by Au NPs and randomly distributed nanoholes. In addition, we simulated a pyramid with nanoholes but without AuNPs (Figure 6j-1) to explore the influence of nanoholes and AuNPs on the electric field. It can be seen that both nanoholes and AuNPs have a certain influence on electric field intensity. However, in the actual structure, the volume of nanoholes or pores is larger than AuNPs. The simulation is a relatively simplified situation and serves as a reference. The nanoholes contribute more to the reflectance, while AuNPs also help to reduce the reflectance. Therefore, it can be inferred that this structure reduces the reflectance, which is the synergistic effect of the inverted pyramidal structure obtained using anisotropic wet etching, the porous structure formed through the MacEtch process and the Au NPs. In addition, this structure not only achieves a reduction in reflectivity, but also has self-cleaning properties. This self-cleaning capability maintains the anti-reflective properties when contaminated with dust particles in an outdoor environment. Figure 7b shows the contact angle (KRÜSS $\mathrm{GmbH}, \mathrm{DSA} 30 \mathrm{E}, \mathrm{Hamburg}$, Germany) of a 5-microliter water drop on an 8-micrometer inverted pyramidal array after being treated with the MacEtch process for 8 min, which exhibits hydrophobicity by increasing the contact angle from 39.3 to $132.4^{\circ}$ compared to 
polished Si wafer (Figure 7a). There is a 2-nanometer unavoidable oxide layer and residual organic matter on polished $\mathrm{Si}$ wafer. Therefore, the inverted pyramids with deeply etched holes can take advantage of the self-cleaning behavior induced by the hydrophobic surface properties in addition to its anti-reflective properties.
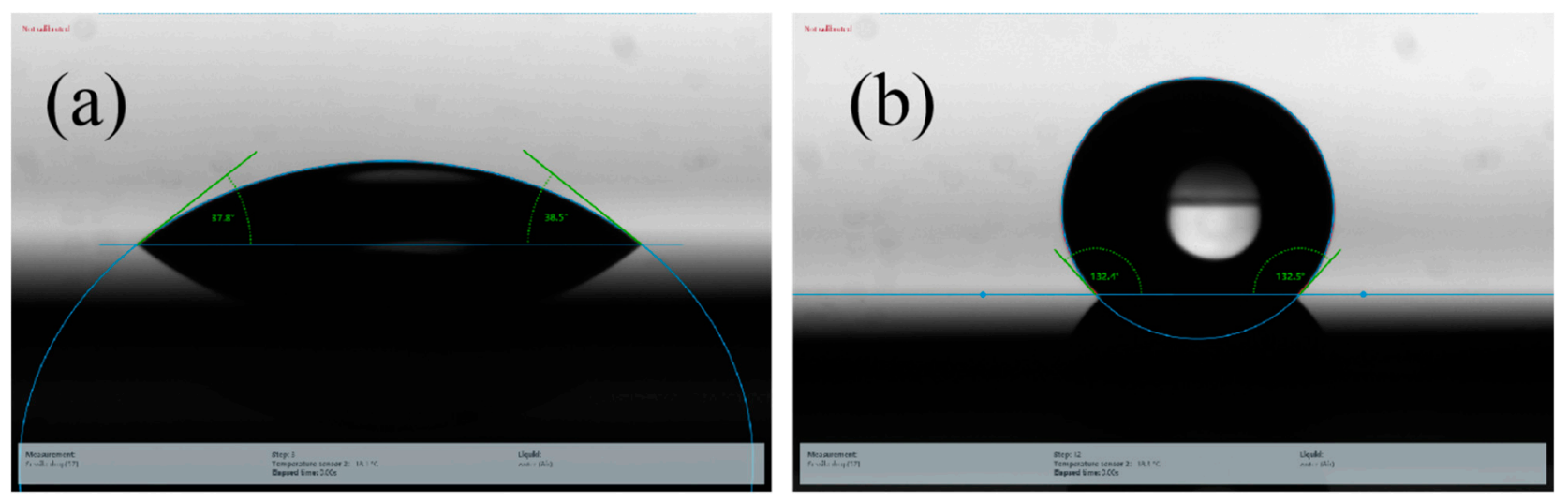

Figure 7. Photographs of the contact angle of a 5-microliter water drop (a) on bare silicone, (b) on an 8-micrometer inverted pyramidal array after being treated with the MacEtch process for $8 \mathrm{~min}$.

\section{Conclusions}

In summary, an inverted pyramidal array with randomly distributed nanoholes was prepared on a silicon substrate using a combination of anisotropic wet etching and MacEtch, which has a strong anti-reflection capability. This morphology facilitates the multiple reflection and absorption of photons, thus improving the reflection efficiency. We investigated the effect of inverted pyramidal structure size and MacEtch time on reflectance. The structure exhibits excellent anti-reflective properties. When the anisotropic wet etching time is $20 \mathrm{~min}$ and the MacEtch time is $8 \mathrm{~min}$, the average reflectance of this structure in the UV region $(250-400 \mathrm{~nm})$, visible region $(400-760 \mathrm{~nm})$ and NIR region $(760-2600 \mathrm{~nm})$ are 1.11, 0.63 and $3.76 \%$, respectively. The resulting nanostructure can achieve the lowest reflectance of $0.45 \%$ at $700 \mathrm{~nm}$ and the highest reflectance of $5.86 \%$ at $2402 \mathrm{~nm}$. The reflectance at broadband wavelength (250-2600 $\mathrm{nm}$ ) is 14.4 and 3.4 times lower than silicon wafer and silicon pyramids. We used FDTD to simulate the electromagnetic enhancement phenomenon and observed the internal electric field. It demonstrated that the distribution of light depends on the designed structure. In particular, such a structure exhibits high hydrophobicity with a contact angle up to $132.4^{\circ}$. We expect that such an efficient fabrication method can find widespread applications in photo detectors sensitive layers for luminescence and other optoelectronic devices.

Author Contributions: Conceptualization, Y.Z. and H.L.; software, Y.Z.; methodology, Y.Z. and K.Z.; writing-original draft preparation, Y.Z.; writing-review, H.L. and C.X. All authors have read and agreed to the published version of the manuscript.

Funding: This research was funded by the National Key Research and Development Program of China (Grant No. 2017YFA0206002); National Natural Science Foundation of China (Grant Nos. 61804169, U1832217, and 61821091).

Data Availability Statement: All data generated or analysed during this study are included in this published article.

Conflicts of Interest: The authors declare no conflict of interest. 


\section{References}

1. Garin, M.; Heinonen, J.; Werner, L.; Pasanen, T.P.; Vahanissi, V.; Haarahiltunen, A.; Juntunen, M.A.; Savin, H. Black-Silicon Ultraviolet Photodiodes Achieve External Quantum Efficiency above 130. Phys. Rev. Lett. 2020, 125, 117702. [CrossRef] [PubMed]

2. Green, M.A.; Bremner, S.P. Energy conversion approaches and materials for high-efficiency photovoltaics. Nat. Mater. 2016, 16, 23-34. [CrossRef] [PubMed]

3. Holst, G.C.; Krapels, K.A.; Teaney, B.P.; Reynolds, J.P. Reflective band image generation in the night vision integrated performance model. Int. Soc. Opt. Photonics 2016, 9820, 982006.

4. Capasso, F.; Tsang, W.T.; Hutchinson, A.L.; Williams, G.F. Enhancement of electron impact ionization in a superlattice: A new avalanche photodiode with a large ionization rate ratio. Appl. Phys. Lett. 1982, 40, 38-40. [CrossRef]

5. Yong, S.T.; Mosset, J.B.; Loude, J.F.; Yong, C.; Morel, C. Characterization of two deep-diffusion avalanche photodiode array prototypes with different optical coatings. IEEE Trans. Nucl. Sci. 2004, 51, 2279-2283.

6. Yao, T.-F.; Wu, P.-H.; Wu, T.-M.; Cheng, C.-W.; Yang, S.-Y. Fabrication of anti-reflective structures using hot embossing with a stainless steel template irradiated by femtosecond laser. Microelectron. Eng. 2011, 88, 2908-2912. [CrossRef]

7. Huang, Z.; Song, X.; Zhong, S.; Xu, H.; Luo, W.; Zhu, X.; Shen, W. 20.0\% Efficiency Si Nano/Microstructures Based Solar Cells with Excellent Broadband Spectral Response. Adv. Funct. Mater. 2016, 26, 1892-1898. [CrossRef]

8. Kanevskii, V.I.; Kolienov, S.O. Analysis of electrodynamic conditions of photo-assisted nanoscale polishing of silica covered with calcium hypochlorite: Theoretical analysis. J. Mod. Opt. 2020, 67, 242-251. [CrossRef]

9. Wang, L.; Chen, K.; Tong, H.; Wang, K.; Tao, L.; Zhang, Y.; Zhou, X. Inverted pyramid Er ${ }^{3+}$ and $\mathrm{Yb}^{3+} \mathrm{Co}_{-\mathrm{doped}} \mathrm{TiO}_{2}$ nanorod arrays based perovskite solar cell: Infrared response and improved current density. Ceram. Int. 2020, 46, 12073-12079. [CrossRef]

10. Yu, M.; Huang, W.; Li, P.; Huang, H.; Zhang, K.; Zhu, X. Morphology evolution of TiO2 nanotubes with additional reducing agent: Evidence of oxygen release. Electrochem. Commun. 2019, 98, 28-32. [CrossRef]

11. Kim, K.; Song, Y.; Oh, J. Nano/micro dual-textured antireflective subwavelength structures in anisotropically etched GaAs. Opt. Lett. 2017, 42, 3105-3108. [CrossRef] [PubMed]

12. Sun, C.-H.; Jiang, P.; Jiang, B. Broadband moth-eye antireflection coatings on silicon. Appl. Phys. Lett. 2008, 92, 061112. [CrossRef]

13. Okabe, T.; Yano, T.; Yatagawa, K.; Taniguchi, J. Polyimide moth-eye nanostructures formed by oxygen ion beam etching for anti-reflection layers. Microelectron. Eng. 2021, 242-243, 111559. [CrossRef]

14. Chen, J.-Y.; Yu, M.-H.; Chang, S.-F.; Wen Sun, K. Highly efficient poly(3,4-ethylenedioxythiophene):poly(styrenesulfonate)/Si hybrid solar cells with imprinted nanopyramid structures. Appl. Phys. Lett. 2013, 103, 133901. [CrossRef]

15. Tsujino, K.; Matsumura, M. Boring Deep Cylindrical Nanoholes in Silicon Using Silver Nanoparticles as a Catalyst. Adv. Mater. 2005, 17, 1045-1047. [CrossRef]

16. Oh, J.; Yuan, H.C.; Branz, H.M. An 18.2\%-efficient black-silicon solar cell achieved through control of carrier recombination in nanostructures. Nat. Nanotechnol. 2012, 7,743-748. [CrossRef]

17. Lv, J.; Zhang, T.; Zhang, P.; Zhao, Y.; Li, S. Review Application of Nanostructured Black Silicon. Nanoscale Res. Lett. 2018, 13, 110. [CrossRef] [PubMed]

18. Yang, Z.; Feng, B.; Lu, B.; Chen, Y.; Li, W.; Zhang, W.; Li, T. A study of nano-structural effect on the polarization characteristics of metallic sub-wavelength grating polarizers in visible wavelengths. Microelectron. Eng. 2020, 227, 111327. [CrossRef]

19. Li, H.; Niu, J.; Zhang, C.; Niu, G.; Ye, X.; Xie, C. Ultra-Broadband High-Efficiency Solar Absorber Based on Double-Size Cross-Shaped Refractory Metals. Nanomaterials 2020, 10, 552. [CrossRef]

20. Gao, K.; Liu, Y.; Fan, Y.; Shi, L.; Zhuang, Y.; Cui, Y.; Yuan, S.; Wan, Y.; Shen, W.; Huang, Z. High-Efficiency Silicon Inverted Pyramid-Based Passivated Emitter and Rear Cells. Nanoscale Res. Lett. 2020, 15, 174. [CrossRef] [PubMed]

21. Papet, P.; Nichiporuk, O.; Kaminski, A.; Rozier, Y.; Kraiem, J.; Lelievre, J.F.; Chaumartin, A.; Fave, A.; Lemiti, M. Pyramidal texturing of silicon solar cell with TMAH chemical anisotropic etching. Sol. Energy Mater. Sol. Cells 2006, 90, 2319-2328. [CrossRef]

22. Lin, Y.-K.; Chen, Y.-S.; Hsueh, C.-H. Combined metal-assisted chemical etching and anisotropic wet etching for anti-reflection inverted pyramidal cavities on dendrite-like textured substrates. Results Phys. 2019, 12, 244-249. [CrossRef]

23. Rahman, T.; Navarro-Cia, M.; Fobelets, K. High density micro-pyramids with silicon nanowire array for photovoltaic applications. Nanotechnology 2014, 25, 485202. [CrossRef]

24. Kalogirou, S.A. Solar thermal collectors and applications. Prog. Energy Combust. Sci. 2004, 30, 231-295. [CrossRef]

25. Razykov, T.M.; Ferekides, C.S.; Morel, D.; Stefanakos, E.; Ullal, H.S.; Upadhyaya, H.M. Solar photovoltaic electricity: Current status and future prospects. Sol. Energy 2011, 85, 1580-1608. [CrossRef]

26. Zhang, Z.; Martinsen, T.; Liu, G.; Tayyib, M.; Cui, D.; Boer, M.J.; Karlsen, F.; Jakobsen, H.; Xue, C.; Wang, K. Ultralow Broadband Reflectivity in Black Silicon via Synergy between Hierarchical Texture and Specific-Size Au Nanoparticles. Adv. Opt. Mater. 2020, 8, 2000668. [CrossRef]

27. Gupta, P.P.A. Chandra Shekhar Sharma, Surface Texturing of Silicon $\{100\}$ in an Extremely Low Concentration TMAH for Minimized Reflectivity. ECS J. Solid State Sci. Technol. 2019, 8, P622-P628. [CrossRef]

28. Campbell, P.; Green, M.A. Light trapping properties of pyramidally textured surfaces. J. Appl. Phys. 1987, 62, 243-249. [CrossRef]

29. Omer, A.A.A.; He, Z.; Hong, S.; Chang, Y.; Yu, J.; Li, S.; Ma, W.; Liu, W.; El Kolaly, W.; Chen, R. Ultra-Thin Silicon Wafers Fabrication and Inverted Pyramid Texturing Based on Cu-Catalyzed Chemical Etching. Silicon 2020, 13, 351-359. [CrossRef]

30. Amalathas, A.P.; Alkaisi, M.M. Efficient light trapping nanopyramid structures for solar cells patterned using UV nanoimprint lithography. Mater. Sci. Semicond. Process. 2017, 57, 54-58. [CrossRef] 
31. Chen, K.; Zha, J.; Hu, F.; Ye, X.; Zou, S.; Vähänissi, V.; Pearce, J.M.; Savin, H.; Su, X. MACE nano-texture process applicable for both single- and multi-crystalline diamond-wire sawn Si solar cells. Sol. Energy Mater. Sol. Cells 2019, 191, 1-8. [CrossRef]

32. Romano, L.; Kagias, M.; Vila-Comamala, J.; Jefimovs, K.; Tseng, L.T.; Guzenko, V.A.; Stampanoni, M. Metal assisted chemical etching of silicon in the gas phase: A nanofabrication platform for X-ray optics. Nanoscale Horiz. 2020, 5, 869-879. [CrossRef]

33. Romano, L.; Stampanoni, M. Microfabrication of X-ray Optics by Metal Assisted Chemical Etching: A Review. Micromachines 2020, 11, 589. [CrossRef]

34. Jiang, B.; Dai, H.; Zhao, Q.; Lin, J.; Chu, L.; Li, Y.; Fu, P.; Wu, G.; Ji, J.; Li, M. The path of mass transfer during Au thin film-assisted chemical etching by designed surface barriers. RSC Adv. 2017, 7, 11522-11527. [CrossRef]

35. Li, H.; Ye, T.; Shi, L.; Xie, C. Fabrication of ultra-high aspect ratio (>160:1) silicon nanostructures by using Au metal assisted chemical etching. J. Micromech. Microeng. 2017, 27, 124002. [CrossRef]

36. Ding, K.; Zhang, M.; Mao, J.; Xiao, P.; Zhang, X.W.; Wu, D.; Zhang, X.J.; Jie, J.S. High-resolution image patterned silicon wafer with inverted pyramid micro-structure arrays for decorative solar cells. Mater. Today Energy 2020, 18, 100493. [CrossRef]

37. Tang, Q.; Yao, H.; Xu, B.; Ge, J. Enhanced energy conversion efficiency of Al-BSF c-Si solar cell by a novel hierarchical structure composed of inverted pyramids with different sizes. Sol. Energy 2020, 208, 1-9. [CrossRef]

38. Markel, V. Introduction to the Maxwell Garnett approximation tutorial. J. Opt. Soc. Am. 2016, 33, 1244-1256. [CrossRef] [PubMed] 